\title{
Analysis of a finite element formulation for modelling phase separation
}

\author{
Garth N. Wells ${ }^{1}$ and Krishna Garikipati ${ }^{2}$ \\ 1 Delft University of Technology g.n.wells@tudelft.nl \\ 2 University of Michigan krishna@umich.edu
}

Summary. The Cahn-Hilliard equation is of importance in materials science and a range of other fields. It represents a diffuse interface model for simulating the evolution of phase separation in solids and fluids, and is a nonlinear fourth-order parabolic equation, which makes its numerical solution particularly challenging. To this end, a finite element formulation has been developed which can solve the CahnHilliard equation in its primal form using $C^{0}$ basis functions. Here, analysis of a fully discrete version of this method is presented in the form of a priori uniqueness, stability and error analysis.

Key words: Cahn-Hilliard equation, discontinuous Galerkin method, phase separation.

\section{Introduction}

The Cahn-Hilliard [1] equation models the separation of phases in binary mixtures. It is particularly relevant in material science, where it describes microstructure evolution in alloys. As a diffuse interface model, it represents the boundaries between pure phases as a small region across which the relative concentration varies rapidly. This is an advantage in that discontinuities at phase boundaries do not have to be modelled explicitly, but comes at the cost of needing to resolve gradients at phase boundaries well and a high degree of mathematical complexity.

The numerical solution of the Cahn-Hilliard equation is particularly challenging on a number of fronts. Foremost in the difficulties is the presence of fourth-order spatial derivatives. A fourth-order term is necessary to balance the presence of a chemical potential in the Cahn-Hilliard equation which is derived from a non-convex free energy function. The non-convex nature of the chemical free energy further complicates both the numerical solution strategy and the analysis of numerical methods. Extensive research has been 
performed over the past 20 years into the mathematical analysis of finite element formulations for the Cahn-Hilliard equation. Initial efforts focused upon $C^{1}[2,3]$ and non-conforming methods [4]. The majority of later efforts focused upon a mixed finite element formulation based on an operator split (see Refs. $[5,6,7,8]$ for a selection of works).

We present here analysis of the finite element formulation for the CahnHilliard equation presented by Wells et al. [9]. The formulation allows the solution of the Cahn-Hilliard equation using standard $C^{0}$ basis functions in the primal form, with continuity of the normal derivative across element boundaries enforced weakly. In Wells et al. [9], stability and convergence in an energy-like norm was demonstrated for the time-continuous case. Here, a fully discrete formulation is analysed and an error estimate in the $L^{2}$ norm is presented.

The rest of this work is organised as follows: the Cahn-Hilliard equation is presented in its strong form, after which the semi-discrete Galerkin formulation is presented and some key results are summarised. The fully discrete formulation is then presented, followed by a priori analysis of uniqueness, stability and error in the $L^{2}$ norm. A numerical example is presented, simulating phase separation in a uniform mixture which is randomly-perturbed. Following the numerical example, conclusions are drawn.

\section{Cahn-Hilliard equation}

Consider a binary mixture and let the concentration of one of its constituents, say $A$, be denoted by $c$ satisfying $0<c<1$. The concentration of the other constituent, $B$, is $1-c$. Pure phases are obtained for $c=0$ and $c=1$. Let the mixture occupy an open, simply connected region in space, $\Omega \subset \mathbb{R}^{d}$, where $d=1,2$ or 3 . The boundary of $\Omega$ is supposed to be sufficiently smooth, and is denoted by $\Gamma=\partial \Omega$, with outward unit normal $\boldsymbol{n}$. In strong form we have the following problem: find $c: \bar{\Omega} \times[0, T] \rightarrow \mathbb{R}$ such that

$$
\begin{array}{ll}
c, t=\nabla \cdot\left(M \nabla\left(\mu-\lambda \nabla^{2} c\right)\right) & \text { in } \Omega \times(0, T), \\
\nabla c \cdot \boldsymbol{n}=0 & \text { on } \Gamma \times(0, T), \\
\nabla\left(\mu-\lambda \nabla^{2} c\right) \cdot \boldsymbol{n}=s & \text { on } \Gamma \times(0, T), \\
c(\boldsymbol{x}, 0)=c_{0}(\boldsymbol{x}) & \text { in } \Omega,
\end{array}
$$

where $M \geq 0$ is the mobility, $\mu$ is the chemical potential, $\lambda>0$ is a constant related to the interfacial energy and $c_{0}(\boldsymbol{x})$ are the initial conditions. The boundary conditions of zero normal derivative for the concentration and zero mass flux on the entire boundary are typical for the Cahn-Hilliard equation. The Cahn-Hilliard equation is a fourth-order diffusion equation, and the presence of fourth-order spatial derivatives is due to the introduction of an expression for the surface free-energy $\Psi^{s}$,

$$
\Psi^{s}-\frac{\lambda}{2} \nabla c \cdot \nabla c .
$$


The mobility $M$ can be either constant or concentration-dependent. If made concentration-dependent, a common choice is

$$
M=c(1-c),
$$

which largely restricts diffusion processes to phase interface zones. The chemical potential in the case of phase separation problems comes from a non-convex chemical free-energy $\Psi^{\mathrm{c}}$. It is the non-convex nature of the free-energy which drives phase separation. A typical chemical free-energy of a solution is given by $[1]$

$$
\Psi^{\mathrm{c}}=N k T(c \ln c+(1-c) \ln (1-c))+N \omega c(1-c),
$$

where $N$ is the number of molecules per unit volume, $k$ is Boltzmann's constant, $T$ is the absolute temperature and $\omega$ is a parameter related to the mixing enthalpy that determines the shape of $\Psi^{\mathrm{c}}$. For $\omega>2 k T$, the chemical free energy is non-convex, with two wells close to $c=0$ and $c=1$ which drives phase segregation into the two binodal points. The potential $\mu$ is given by the functional derivative of the chemical free-energy with respect to the concentration, $D_{c} \Psi^{c}$. Another possibility is the use of a quartic polynomial for the chemical free energy,

$$
\Psi^{c}=\frac{1}{4} c^{2}(1-c)^{2},
$$

which has the advantage of being continuous on the real line. This simplifies the analysis of formulations and leads to more robust numerical procedures. It does however allow for solutions outside of the range $[0,1]$. The term $\lambda$ governs the magnitude of the free-energy related the interfaces in the presence of a given concentration gradient.

\section{Semi-discrete Galerkin formulation}

\subsection{Definitions}

Consider a partition of $\Omega$ into $n_{\mathrm{el}}$ polygonal open sets, $\Omega_{e}$, each with boundary $\Gamma_{e}=\partial \Omega_{e}$ :

$$
\Omega=\bigcup_{e=1}^{\overline{n_{\mathrm{el}}} \Omega_{e}}, \quad \text { such that } \bigcap_{e=1}^{n_{\mathrm{el}}} \Omega_{e}=\emptyset .
$$

It is assumed that $\Omega$ is a polygon and hence can be partitioned exactly. The union of inter-element boundaries and the boundary $\Gamma$ is denoted by

$$
\widetilde{\Gamma}=\bigcup_{e=1}^{n_{\mathrm{el}}} \Gamma_{e}
$$

where $\Gamma_{e}=\partial \Omega_{e}$, and the union of element interiors is denoted by 


$$
\widetilde{\Omega}=\bigcup_{e=1}^{n_{\mathrm{el}}} \Omega_{e}
$$

The jump operator for a vector is denoted by

$$
\begin{aligned}
& \llbracket \boldsymbol{a} \rrbracket=\boldsymbol{a}_{1} \cdot \boldsymbol{n}_{1}+\boldsymbol{a}_{2} \cdot \boldsymbol{n}_{2}, \quad \text { on } \widetilde{\Gamma} \backslash \Gamma, \\
& \llbracket \boldsymbol{a} \rrbracket=\boldsymbol{a} \cdot \boldsymbol{n} \quad \text { on } \Gamma,
\end{aligned}
$$

where the subscripts refer to the face of the element on either side of each inter-element boundary, and $\boldsymbol{n}$ is the unit outward normal to an element boundary. The average operator is denoted by

$$
\begin{aligned}
& \langle a\rangle=\frac{1}{2}\left(a_{1}+a_{2}\right) \quad \widetilde{\Gamma} \backslash \Gamma, \\
& \langle a\rangle=a \quad \text { on } \Gamma,
\end{aligned}
$$

where again the subscripts refer to the face of the element on either side of each inter-element boundary.

\subsection{Semi-discrete formulation}

Classical Galerkin methods for the Cahn-Hilliard equation with the considered boundary conditions seek approximate solutions in a subspace of $H_{E}^{2}(\Omega)$, which is defined as

$$
H_{E}^{2}(\Omega)=\left\{c^{h} \mid c^{h} \in H^{2}(\Omega), \nabla c^{h} \cdot \boldsymbol{n} \text { on } \Gamma\right\} .
$$

The space satisfies the considered Dirichlet boundary condition by construction. However, in a finite element context, such functions are difficult to construct. Here, a Galerkin formulation for the Cahn-Hilliard equation is examined which looks for solutions in a subspace of $H^{1}(\Omega)$, thereby allowing the use of standard $C^{0}$ Lagrange shape functions. Consider therefore the function space

$$
W^{h}=\left\{c^{h} \mid c^{h} \in H^{1}(\Omega), c^{h} \in P^{k}\left(\Omega_{e}\right) \forall e\right\},
$$

where $P^{k}\left(\Omega_{e}\right)$ is the space of the standard polynomial finite element shape functions on element $\Omega_{e}$ and $k$ is the polynomial order. Note that $c^{h} \in$ $H^{2}(\widetilde{\Omega})$. A finite element problem for the Cahn-Hilliard equation then involves [9]: find $c^{h}(t) \in W^{h}, t \in[0, T]$ such that

$$
\left(w^{h}, \dot{c}^{h}\right)_{\Omega}+a\left(w^{h}, c^{h}\right)=0 \quad \forall w^{h} \in W^{h},
$$

where

$$
\begin{aligned}
a\left(w^{h}, c^{h}\right)_{\Omega} & =\left(\nabla w^{h}, M^{h} \nabla \mu^{h}\right)_{\Omega}+\left(\nabla^{2} w^{h}, M^{h} \lambda \nabla^{2} c^{h}\right)_{\widetilde{\Omega}} \\
+ & \left(\nabla w^{h},\left(\nabla M^{h}\right) \lambda \nabla^{2} c^{h}\right)_{\widetilde{\Omega}}-\left(\llbracket \nabla w^{h} \rrbracket,\left\langle M^{h} \lambda \nabla^{2} c^{h}\right\rangle\right)_{\widetilde{\Gamma}} \\
& \left.-\left(\left\langle M^{h} \lambda \nabla^{2} w^{h}\right\rangle, \llbracket \nabla c^{h} \rrbracket\right)_{\widetilde{\Gamma}}+\frac{\alpha}{h}\left(\llbracket \nabla w^{h} \rrbracket, M^{h} \lambda \llbracket \nabla c^{h} \rrbracket\right)\right)_{\widetilde{\Gamma}},
\end{aligned}
$$


and $\alpha$ is a dimensionless penalty term and $h$ is a measure of the element size. The notation

$$
(a, b)_{X}=\int_{X} a b d X
$$

for inner products has been adopted. In effect, the formulation imposes continuity of (a) the normal derivative of the concentration, (b) the normal flux and (c) the Laplacian of the concentration across element boundaries in a weak sense. For simplicity of notation, it has been assumed that $\alpha$ and $h$ are constant for all elements.

The formulation can be shown to be consistent with the Cahn-Hilliard equation, and is stable if the penalty term is chosen to be sufficiently large [9]. How large it must be is dependent on constants in various inequalities which are related to the order of elements and the element geometry. Stability estimates and a priori error estimates in an energy-like norm for the semi-discrete problem can be found in Wells et al. [9]. The focus in the rest of this work is on estimates for the fully discrete problem.

\section{Fully discrete formulation}

We consider now the stability of a time-discrete problem whose numerical scheme is parametrised by $\theta \in[0,1]$. The time continuous problem in equation (18) is replaced by a sequence of discrete steps at $t_{1}, t_{2}, \cdots, t_{n}, t_{n+1}$, where $\Delta t=t_{n+1}-t_{n}$. The problem at $t_{n+1}$ then becomes: find $c^{h, n+1} \in W^{h}$ such that

$$
\left(w^{h}, \frac{c^{h, n+1}-c^{h, n}}{\Delta t}\right)+a\left(w^{h}, c^{h, n+\theta}\right)=0 \quad \forall c^{h} \in W^{h},
$$

where

$$
c^{h, n+\theta}=(1-\theta) c^{h, n}+\theta c^{h, n+1} .
$$

As usual, $\theta=0$ leads to the forward Euler scheme, $\theta=1$ leads to the backward Euler scheme and $\theta=1 / 2$ leads to the Crank-Nicolson method.

In the following analysis, we restrict ourselves to the case of constant mobility $(M=1)$ and a continuously differentiable chemical potential with the property

$$
\frac{d \mu}{d c} \geq-b_{0},
$$

where $b_{0} \geq 0$. This condition holds for all commonly adopted chemical potentials.

\subsection{Uniqueness}

Consider the function $\beta=c_{1}^{h, n+1}-c_{2}^{h, n+1}$, where $c_{1}^{h, n+1}$ and $c_{2}^{h, n+1}$ are solutions to the fully discrete problem. Inserting $\beta$ into the right-hand slot of 
the forms in equation (21), and noting the $w^{h}=\beta$ is an admissible weighting function,

$$
\begin{aligned}
\|\beta\|_{\Omega}^{2}+\Delta t \theta \lambda\left\|\nabla^{2} \beta\right\|_{\widetilde{\Omega}}^{2} & -2 \Delta t \theta\left(\llbracket \nabla \beta \rrbracket,\left\langle\nabla^{2} \beta\right\rangle\right)_{\widetilde{\Gamma}}+\Delta t \theta \frac{\alpha \lambda}{h}\|\llbracket \nabla \beta \rrbracket\|_{\widetilde{\Gamma}}^{2} \\
& =-\Delta t\left(\nabla \beta, \nabla\left(\mu\left(c_{1}^{h, n+\theta}\right)-\mu\left(c_{2}^{h, n+\theta}\right)\right)\right)_{\Omega},
\end{aligned}
$$

where $\|a\|_{X}$ indicates the $L^{2}$ norm on the domain $X$. From integration by parts,

$$
\begin{aligned}
& \left|\left(\nabla \beta, \nabla\left(\mu\left(c_{1}^{h, n+\theta}\right)-\mu\left(c_{2}^{h, n+\theta}\right)\right)\right)_{\Omega}\right| \\
& \leq\left|\left(\nabla^{2} \beta, \mu\left(c_{1}^{h, n+\theta}\right)-\mu\left({ }^{h, n+\theta} c_{2}\right)\right)_{\widetilde{\Omega}}\right| \\
& \quad+\left|\left(\llbracket \nabla \beta \rrbracket, \mu\left(c_{1}^{h, n+\theta}\right)-\mu\left(c_{2}^{h, n+\theta}\right)\right)_{\widetilde{\Gamma}}\right|
\end{aligned}
$$

together with Lipschitz continuity of the chemical potential,

$$
\begin{aligned}
\left|\mu\left(c_{1}^{h, n+\theta}\right)-\mu\left(c_{2}^{h, n+\theta}\right)\right| & \leq L\left|c_{1}^{h, n+\theta}-c_{2}^{h, n+\theta}\right| \\
& =L \theta|\beta|,
\end{aligned}
$$

where $L>0$, and the Cauchy-Schwartz inequality, it follows that:

$$
\begin{aligned}
\mid\left(\nabla \beta, \nabla\left(\mu\left(c_{1}^{h, n+\theta}\right)-\mu\right.\right. & \left.\left.\left(c_{2}^{h, n+\theta}\right)\right)\right)_{\Omega} \mid \\
& \leq \theta L\left\|\nabla^{2} \beta\right\|_{\widetilde{\Omega}}\|\beta\|_{\Omega}+\theta L\|\llbracket \nabla \beta \rrbracket\|_{\widetilde{\Gamma}}\|\beta\|_{\widetilde{\Gamma}} .
\end{aligned}
$$

Application of Young's inequality then leads to:

$$
\begin{aligned}
\mid(\nabla \beta, \nabla & \left.\left(\mu\left(c_{1}^{h, n+\theta}\right)-\mu\left(c_{2}^{h, n+\theta}\right)\right)\right)_{\Omega} \mid \\
& \leq \frac{\theta L}{2 \epsilon_{0}}\left\|\nabla^{2} \beta\right\|_{\widetilde{\Omega}}^{2}+\frac{\theta L \epsilon_{0}}{2}\|\beta\|_{\Omega}+\frac{\theta L}{2 \epsilon_{1}}\|\llbracket \nabla \beta \rrbracket\|_{\widetilde{\Gamma}}^{2}+\frac{\theta L \epsilon_{1}}{2}\|\beta\|_{\widetilde{\Gamma}}^{2},
\end{aligned}
$$

where $\epsilon_{0}, \epsilon_{1}>0$. Turning attention now to the first inter-element term in equation (24),

$$
2\left|(\llbracket \nabla \beta \rrbracket,\langle\nabla \beta\rangle)_{\widetilde{\Gamma}}\right| \leq \epsilon_{2} C\left\|\nabla^{2} \beta\right\|_{\widetilde{\Omega}}^{2}+\frac{1}{h \epsilon_{2}}\|\llbracket \nabla \beta \rrbracket\|_{\widetilde{\Gamma}}^{2} .
$$

For the derivation of this expression, we refer the reader to Wells et al. [9]. Also from Wells et al. [9], there exists $C>0$ such that

$$
\|\beta\|_{\widetilde{\Gamma}}^{2} \leq \frac{C}{h}\|\beta\|_{\Omega}^{2}
$$

Using these results in equation (24), it follows that 


$$
\begin{aligned}
\|\beta\|_{\Omega}^{2}+\Delta t & \theta \lambda\left\|\nabla^{2} \beta\right\|_{\widetilde{\Omega}}^{2}+\Delta t \theta \frac{\alpha \lambda}{h}\|\llbracket \nabla \beta \rrbracket\|_{\widetilde{\Gamma}}^{2} \\
\leq & \frac{\Delta t \theta L}{2 \epsilon_{0}}\left\|\nabla^{2} \beta\right\|_{\widetilde{\Omega}}^{2}+\frac{\Delta t \theta L \epsilon_{0}}{2}\|\beta\|_{\Omega}^{2}+\frac{\Delta t \theta L}{2 \epsilon_{1}}\|\llbracket \nabla \beta \rrbracket\|_{\widetilde{\Gamma}}^{2} \\
& +\frac{\Delta t \theta L \epsilon_{1} C}{2 h}\|\beta\|_{\Omega}^{2}+\Delta t \theta \lambda \epsilon_{2} C\left\|\nabla^{2} \beta\right\|_{\widetilde{\Omega}}^{2}+\frac{\Delta t \theta \lambda}{h \epsilon_{2}}\|\llbracket \nabla \beta \rrbracket\|_{\widetilde{\Gamma}}^{2} .
\end{aligned}
$$

Grouping related terms together,

$$
\begin{aligned}
\|\beta\|_{\Omega}^{2}+(\Delta t \theta \lambda & \left.-\frac{\Delta t \theta L}{2 \epsilon_{0}}-\lambda \Delta t \theta C \epsilon_{2}\right)\left\|\nabla^{2} \beta\right\|_{\widetilde{\Omega}}^{2} \\
+ & \left(\frac{\Delta t \theta \alpha \lambda}{h}-\frac{\Delta t \theta L}{2 \epsilon_{1}}-\frac{\Delta t \theta \lambda}{h \epsilon_{2}}\right)\|\llbracket \nabla \beta \rrbracket\|_{\widetilde{\Gamma}}^{2} \\
\leq & \leq\left(\frac{\Delta t \theta L \epsilon_{0}}{2}+\frac{\Delta t \theta L \epsilon_{1} C}{2 h}\right)\|\beta\|_{\Omega}^{2} .
\end{aligned}
$$

The goal now is to select $\epsilon_{i}$ such that the RHS is greater than $\|\beta\|_{\Omega}^{2}$ and all terms on the LHS are positive. Setting $\epsilon_{0}=1 / \Delta t \theta L, \epsilon_{1}=h / \Delta t \theta L C$ and $\epsilon_{2}=\epsilon_{2}^{\prime} / C$

$$
\begin{aligned}
\|\beta\|_{\Omega}^{2}+\theta(\Delta t \lambda & \left.-\frac{\Delta t^{2} \theta L^{2}}{2}-\lambda \Delta t \epsilon_{2}^{\prime}\right)\left\|\nabla^{2} \beta\right\|_{\widetilde{\Omega}}^{2} \\
& +\theta\left(\frac{\Delta t \alpha \lambda}{h}-\frac{\Delta t^{2} \theta L^{2} C}{2 h}-\frac{\Delta t \lambda C}{h \epsilon_{2}^{\prime}}\right)\|\llbracket \nabla \beta \rrbracket\|_{\widetilde{\Gamma}}^{2} \leq\|\beta\|_{\Omega}^{2} .
\end{aligned}
$$

To demonstrate uniqueness, $\Delta t$ and $\alpha$ must be chosen such that all terms on the LHS are greater than or equal to zero. Consider therefore the restriction on the time step

$$
\Delta t<\frac{2 \lambda\left(1-\epsilon_{2}^{\prime}\right)}{\theta L^{2}}
$$

where $0<\epsilon_{2}^{\prime}<1$. Subject to this time step restriction and if

$$
\alpha>\left(1-\epsilon_{2}^{\prime}\right) C+\frac{C}{\epsilon_{2}^{\prime}}
$$

it follows that

$$
\|\beta\|_{\Omega}^{2}+a_{1}\left\|\nabla^{2} \beta\right\|_{\widetilde{\Omega}}^{2}+a_{2}\|\llbracket \nabla \beta \rrbracket\|_{\widetilde{\Gamma}}^{2} \leq\|\beta\|_{\Omega}^{2},
$$

where $a_{1}, a_{2}>0$, which can hold only if $\beta=0$. Therefore, under the time step restriction, and for a sufficiently large penalty, the fully discrete scheme has a unique solution. The analysis indicates that a larger penalty allows for a larger time step since a large $\alpha$ allows for a small $\epsilon_{2}^{\prime}$. Note that the time step restriction is not dependent on the element size $h$. It is a function of model and time stepping parameters only, whereas $\alpha$ is dependent only on element shape parameters and not model parameters.

The time step restriction for uniqueness is due to the non-convex nature of $\Psi^{c}$. It is possible that a more subtle analysis of the problem may lead to a tighter bound for the maximum allowable time step. 


\subsection{Stability}

Stability of the time discrete formulation is now considered by setting $w^{h}=$ $c^{h, n+\theta}$ in equation (21),

$$
\left(c^{h, n+\theta}, \frac{c^{h, n+1}-c^{h, n+1}}{\Delta t}\right)_{\Omega}+a\left(c^{h, n+\theta}, c^{h, n+\theta}\right)=0 .
$$

Noting that

$$
c^{h, n+\theta}=\left(\theta-\frac{1}{2}\right)\left(c^{h, n+1}-c^{h, n}\right)+\frac{c^{h, n+1}+c^{h, n}}{2},
$$

equation (37) can be expressed as:

$$
\begin{aligned}
\left(\theta-\frac{1}{2}\right)\left\|c^{h, n+1}-c^{h, n}\right\|_{\Omega}^{2}+\frac{\left\|c^{h, n+1}\right\|_{\Omega}^{2}-\left\|c^{h, n}\right\|_{\Omega}^{2}}{2 \Delta t} & +a\left(c^{h, n+\theta}, c^{h, n+\theta}\right)=0 .
\end{aligned}
$$

For the case $\theta \in[1 / 2,1]$,

$$
\frac{\left\|c^{h, n+1}\right\|_{\Omega}^{2}-\left\|c^{h, n}\right\|_{\Omega}^{2}}{2 \Delta t}+a\left(c^{h, n+\theta}, c^{h, n+\theta}\right) \leq 0 .
$$

For standard parabolic differential equations, $a\left(c^{h, n+\theta}, c^{h, n+\theta}\right) \geq 0$, leading trivially to $\left\|c^{h, n+1}\right\|^{2} \leq\left\|c^{h, n}\right\|^{2}$ which implies stability. However, this is not the case for the Cahn-Hilliard equation as the term $a\left(c^{h, n+\theta}, c^{h, n+\theta}\right)$ may be negative. Demonstrating stability requires a more subtle approach.

Expanding the term $a\left(c^{h, n+\theta}, c^{h, n+\theta}\right)$ and rearranging equation (40),

$$
\begin{gathered}
\frac{1}{2 \Delta t}\left\|c^{h, n+1}\right\|_{\Omega}^{2}+\lambda\left\|\nabla^{2} c^{h, n+\theta}\right\|_{\widetilde{\Omega}}^{2}+\frac{\alpha \lambda}{h}\left\|\llbracket \nabla c^{h, n+\theta} \rrbracket\right\|_{\widetilde{\Gamma}}^{2} \\
\leq \frac{b_{0}}{2 \epsilon_{0}}\left\|\nabla^{2} c^{h, n+\theta}\right\|_{\widetilde{\Omega}}^{2}+\frac{b_{0}}{2 \epsilon_{1}}\left\|\llbracket \nabla c^{h, n+\theta} \rrbracket\right\|_{\widetilde{\Gamma}}^{2}+\frac{b_{0} \epsilon_{0}}{2}\left\|c^{h, n+\theta}\right\|_{\Omega}^{2} \\
+\frac{b_{0} C \epsilon_{1}}{2 h}\left\|c^{h, n+\theta}\right\|_{\Omega}^{2}+\lambda \epsilon_{2} C\left\|\nabla^{2} c^{h, n+\theta}\right\|_{\widetilde{\Omega}}^{2} \\
+\frac{\lambda}{h \epsilon_{2}}\left\|\llbracket \nabla c^{h, n+\theta} \rrbracket\right\|_{\widetilde{\Gamma}}^{2}+\frac{1}{2 \Delta t}\left\|c^{h, n}\right\|_{\Omega}^{2},
\end{gathered}
$$

where $\epsilon_{i}>0$. Setting now $\epsilon_{0}=b_{0} / 2 \lambda \epsilon_{0}^{\prime}, \epsilon_{1}=b_{0} h / 2 C \lambda \epsilon_{1}^{\prime}$, and $\epsilon_{2}=\epsilon_{2}^{\prime} / C$, it follows that

$$
\begin{aligned}
\frac{1}{2 \Delta t}\left\|c^{h, n+1}\right\|_{\Omega}^{2}+ & \lambda\left(1-\epsilon_{0}^{\prime}-\epsilon_{1}^{\prime}\right)\left\|\nabla^{2} c^{h, n+\theta}\right\|_{\widetilde{\Omega}}^{2} \\
+ & \frac{\lambda}{h}\left(\alpha-C \epsilon_{1}^{\prime}-\frac{C}{\epsilon_{2}^{\prime}}\right)\left\|\llbracket \nabla c^{h, n+\theta} \rrbracket\right\|_{\widetilde{\Gamma}}^{2} \\
& \leq\left(\frac{b^{2}}{4 \lambda \epsilon_{0}^{\prime}}+\frac{b^{2}}{4 \lambda \epsilon_{1}^{\prime}}\right)\left\|c^{h, n+\theta}\right\|_{\Omega}^{2}+\frac{1}{2 \Delta t}\left\|c^{h, n}\right\|_{\Omega}^{2} .
\end{aligned}
$$


Setting $\epsilon_{0}^{\prime}=\epsilon_{1}^{\prime}=1-\epsilon_{2}^{\prime}$, the term $\lambda\left(1-\epsilon_{0}^{\prime}-\epsilon_{1}^{\prime}\right)\left\|\nabla^{2} c^{h, n+\theta}\right\|_{\widetilde{\Omega}}^{2}$ vanishes, and if

$$
\alpha>\left(1-\epsilon_{2}^{\prime}\right) C+\frac{C}{\epsilon_{2}^{\prime}}
$$

then $(\lambda / h)\left(\alpha-C \epsilon_{1}^{\prime}-C / \epsilon_{2}^{\prime}\right)\left\|\llbracket \nabla c^{h, n+\theta} \rrbracket\right\|_{\widetilde{\Gamma}}^{2}>0$. It follows then from equation (42) that

$$
\left\|c^{h, n+1}\right\|_{\Omega}^{2} \leq \frac{\Delta t b^{2}}{\lambda\left(1-\epsilon_{2}^{\prime}\right)}\left\|c^{h, n+\theta}\right\|_{\Omega}^{2}+\left\|c^{h, n}\right\|_{\Omega}^{2} .
$$

For the case $\theta=1$, this implies that if

$$
\Delta t<\frac{\lambda\left(1-\epsilon_{2}^{\prime}\right)}{b^{2}}
$$

then the method is stable as the solution at time step $n+1$ is bounded in terms of the solution at time step $n$, although it does not imply $\left\|c^{h, n+1}\right\|_{\Omega} \leq$ $\left\|c^{h, n}\right\|_{\Omega}$. For other cases,

$$
\left\|c^{h, n+\theta}\right\|_{\Omega}^{2} \leq 2\left(\left\|(1-\theta) c^{h, n}\right\|_{\Omega}^{2}+\left\|\theta c^{h, n+1}\right\|_{\Omega}^{2}\right)
$$

Therefore, for $\theta=1 / 2$ the critical time step is the same as for the $\theta=1$ case. Stability is assured if the critical time step is met since when summing over all time steps, the solution remains bounded in terms of the initial conditions.

Due to the presence of second-order derivatives in the weak form, for the case $\theta \in[0,1 / 2)$ the allowable time step $\Delta t \propto h^{4}$. This makes the usefulness of such schemes extremely limited. Hence, the analysis of such schemes is not pursued here.

\subsection{Accuracy}

Consider the elliptic projection $P^{h} u: H_{E}^{2}(\Omega) \rightarrow W^{h}$ defined by: given $u \in$ $H_{E}^{2}(\Omega)$, find $P^{h} u \in W^{h}$ such that

$$
\begin{aligned}
&\left(\nabla^{2} w^{h}, \nabla^{2}\left(P^{h} u-u\right)\right)_{\widetilde{\Omega}}-\left(\llbracket \nabla w^{h} \rrbracket,\left\langle\nabla^{2}\left(P^{h} u-u\right)\right\rangle\right)_{\widetilde{\Gamma}} \\
&-\left(\left\langle\nabla^{2} w^{h}\right\rangle, \llbracket \nabla^{2}\left(P^{h} u-u\right) \rrbracket\right)_{\widetilde{\Gamma}} \\
& \quad+\frac{\alpha}{h}\left(\llbracket \nabla w^{h} \rrbracket, \llbracket \nabla P^{h} u \rrbracket\right)_{\widetilde{\Gamma}}=0 \quad \forall w^{h} \in W^{h},
\end{aligned}
$$

where

$$
\left(w^{h}, 1\right)_{\Omega}=0, \quad\left(1, P^{h} u\right)_{\Omega}=(1, u)_{\Omega}
$$

It is assumed under these conditions and subject to a suitably large penalty that the solution of equation (47) is unique (see Elliott and French [3] for details). The problem in equation (47) is in essence the same as the problem 
presented in Engel et al. [10], for which error estimates were presented. From these estimates, for $k=2$,

$$
\left\|P^{h} u-u\right\|_{\Omega} \leq C h^{2}\|u\|_{3, \Omega},
$$

and for $k>2$

$$
\left\|P^{h} u-u\right\|_{\Omega} \leq C h^{k+1}\|u\|_{k+1, \Omega} .
$$

The error in the solution at time $t^{n}$ is given by

$$
c^{h, n}-c\left(t_{n}\right)=\underbrace{c^{h, n}-P^{h} c\left(t^{n}\right)}_{e^{h, n}}+\underbrace{P^{h} c\left(t^{n}\right)-c\left(t_{n}\right)}_{\rho^{n}},
$$

where we have an estimate for $\rho^{n}$, therefore we seek to estimate $e^{h, n}$ in order to bound the error.

From equation (21) and consistency of the formulation,

$$
\begin{gathered}
\left(w, \frac{e^{h, n+1}-e^{h, n}}{\Delta t}\right)_{\Omega}+\lambda\left(\nabla^{2} w, \nabla^{2} e^{h, n+\theta}\right)_{\widetilde{\Omega}}+\frac{\alpha \lambda}{h}\left(\llbracket \nabla w \rrbracket, \llbracket \nabla e^{h, n+\theta} \rrbracket\right)_{\widetilde{\Gamma}} \\
=-\left(\nabla w^{h}, \nabla \mu\left(c^{h, n+\theta}\right)-\nabla \mu\left(c\left(t^{n+\theta}\right)\right)\right)_{\Omega} \\
-\left(w, \frac{P^{h} c\left(t^{n+1}\right)-P^{h} c\left(t^{n}\right)}{\Delta t}-c_{, t}\left(t^{n+\theta}\right)\right)_{\Omega} \\
-\lambda\left(\nabla^{2} w^{h}, \nabla^{2}\left((1-\theta) c\left(t^{n}\right)+\theta c\left(t^{n+1}\right)-c\left(t^{n+\theta}\right)\right)\right)_{\widetilde{\Omega}} \\
+\lambda\left(\llbracket \nabla w^{h} \rrbracket,\left\langle\nabla^{2} e^{h, n+\theta}\right\rangle\right)_{\widetilde{\Gamma}}+\lambda\left(\left\langle\nabla^{2} w^{h}\right\rangle, \llbracket \nabla e^{h, n+\theta} \rrbracket\right)_{\widetilde{\Gamma}} \\
+\lambda\left(\llbracket \nabla w^{h} \rrbracket,\left\langle\nabla^{2}\left((1-\theta) c\left(t^{n}\right)+\theta c\left(t^{n+1}\right)-c\left(t^{n+\theta}\right)\right)\right\rangle\right)_{\widetilde{\Gamma}} .
\end{gathered}
$$

We set $w^{h}=e^{h, n+\theta}$ in this relation, and consider $\alpha$ sufficiently large such that $\exists C^{\star}>0$ such that $\forall w^{h} \in W^{h}$

$$
\begin{array}{r}
\left(\nabla^{2} w^{h}, \nabla^{2} w^{h}\right)_{\widetilde{\Omega}}-2\left(\llbracket \nabla w^{h} \rrbracket,\left\langle\nabla^{2} w^{h}\right\rangle\right)_{\widetilde{\Gamma}}+\frac{\alpha}{h}\left(\llbracket \nabla w^{h} \rrbracket, \llbracket \nabla w^{h} \rrbracket\right)_{\widetilde{\Gamma}} \\
\geq C^{\star}\left(\left\|\nabla^{2} w^{h}\right\|_{\widetilde{\Omega}}^{2}+\frac{\alpha}{h}\left\|\llbracket \nabla w^{h} \rrbracket\right\|_{\widetilde{\Gamma}}^{2}\right)
\end{array}
$$

(which is effectively stability of the formulation). This leads to

$$
\begin{gathered}
\left(e^{h, n+\theta}, \frac{e^{h, n+1}-e^{h, n}}{\Delta t}\right)_{\Omega}+C^{\star} \lambda\left\|\nabla^{2} e^{h, n+\theta}\right\|_{\widetilde{\Omega}}^{2}+C^{\star} \frac{\alpha \lambda}{h}\left\|\llbracket \nabla e^{h, n+\theta} \rrbracket\right\|_{\widetilde{\Gamma}}^{2} \\
\leq\left\|\mu\left(c^{h, n+\theta}\right)-\mu\left(c\left(t^{n+\theta}\right)\right)\right\|_{\Omega}\left\|\nabla^{2} e^{h, n+\theta}\right\|_{\widetilde{\Omega}} \\
+\frac{C}{h}\left\|\mu\left(c^{h, n+\theta}\right)-\mu\left(c\left(t^{n+\theta}\right)\right)\right\|_{\Omega}\left\|\llbracket \nabla e^{h, n+\theta} \rrbracket\right\|_{\widetilde{\Gamma}} \\
+\left\|\frac{P^{h} c\left(t^{n+1}\right)-P^{h} c\left(t^{n}\right)}{\Delta t}-c_{, t}\left(t^{n+\theta}\right)\right\|_{\Omega}\left\|e^{h, n+\theta}\right\|_{\Omega} \\
+\lambda\left\|\nabla^{2}\left((1-\theta) c\left(t^{n}\right)+\theta c\left(t^{n+1}\right)-c\left(t^{n+\theta}\right)\right)\right\|\left\|\nabla^{2} e^{h, n+\theta}\right\|_{\widetilde{\Omega}} \\
+\lambda\left\|\llbracket \nabla e^{h, n+\theta} \rrbracket\right\|_{\widetilde{\Gamma}}\left\|\left\langle\nabla^{2}\left((1-\theta) c\left(t^{n}\right)+\theta c\left(t^{n+1}\right)-c\left(t^{n+\theta}\right)\right)\right\rangle\right\|_{\widetilde{\Gamma}} .
\end{gathered}
$$


Using equation (30), the above can be rearranged such that

$$
\begin{aligned}
& \left(e^{h, n+\theta}, \frac{e^{h, n+1}-e^{h, n}}{\Delta t}\right)_{\Omega}+C^{\star} \lambda\left\|\nabla^{2} e^{h, n+\theta}\right\|_{\widetilde{\Omega}}^{2}+C^{\star} \frac{\alpha \lambda}{h}\left\|\llbracket \nabla e^{h, n+\theta} \rrbracket\right\|_{\widetilde{\Gamma}}^{2} \\
\leq & {\left[C\left\|\mu\left(c^{h, n+\theta}\right)-\mu\left(c\left(t^{n+\theta}\right)\right)\right\|_{\Omega}+\left\|\frac{P^{h} c\left(t^{n+1}\right)-P^{h} c\left(t^{n}\right)}{\Delta t}-c_{, t}\left(t^{n+\theta}\right)\right\|_{\Omega}\right.} \\
\left.+(1+C) \lambda\left\|\nabla^{2}\left((1-\theta) c\left(t^{n}\right)+\theta c\left(t^{n+1}\right)-c\left(t^{n+\theta}\right)\right)\right\|_{\Omega}\right] & \left(\left\|\nabla^{2} e^{h, n+\theta}\right\|_{\widetilde{\Omega}}+\frac{1}{h}\left\|\llbracket \nabla e^{h, n+\theta} \rrbracket\right\|_{\widetilde{\Gamma}}\right) \cdot
\end{aligned}
$$

In this form, together with the results in equations (49) and (50), the analysis of Elliott and French [3, Theorem 3.1] can be applied directly, yielding the estimate

$$
\left\|c^{h, n}-c\left(t_{n}\right)\right\|_{\Omega} \leq C\left(h^{p}+\Delta t^{q}\right),
$$

where $C$ is dependent on the exact solution. For $k=2$ gives $p=2$, and $k>2$ gives $p=k+1$. For $\theta \in(1 / 2,1]$ leads to $q=1$ and $\theta=1 / 2$ leads to $q=2$.

\section{Numerical example of phase separation}

The numerical example presented in this section illustrates a response which is typical for the Cahn-Hilliard equation. Phase separation is modelled on a unit square from an initially uniform state which is randomly perturbed. The parameters for the example are given in non-dimensional form. Consider therefore a length scale $L_{0}$, which is representative of the size of the domain $\Omega$, and time scale $T_{0}=L_{0}^{4} / D \lambda$. Relevant dimensionless quantities, denoted with an asterisk, are given by:

$$
t^{\star}=t / T_{0}, \quad x^{\star}=x / L_{0}, \quad \mu_{c}^{\star}=\mu_{c} L_{0}^{2} / \lambda .
$$

Using these, the dimensionless counterpart of equation (1) is given by:

$$
c_{, t^{\star}}=\nabla^{\star} \cdot \beta \nabla^{\star}\left(\mu_{c}^{\star}-\nabla^{\star 2} c\right),
$$

where $\beta$ is a dimensionless term reflecting the nature of the mobility. In the case of constant mobility $\beta=1$, and in case of degenerate mobility $\beta=$ $c(1-c)$.

For this test, the following parameters have been adopted: $\omega / k T=3$, $N k T L_{0}^{2} / \lambda=3000, \beta=c(1-c)$ (degenerate mobility), $\alpha=5$ and $\Delta t^{*}=$ $2 \times 10^{-8}$. For the initial conditions, the average concentration is equal to 0.63 , with random fluctuations of zero mean and no fluctuation greater than 0.05 . Triangular elements with quadratic basis functions $(k=2)$ have been used and the problem has been stepped in time using the Crank-Nicolson method and a full Newton procedure. 


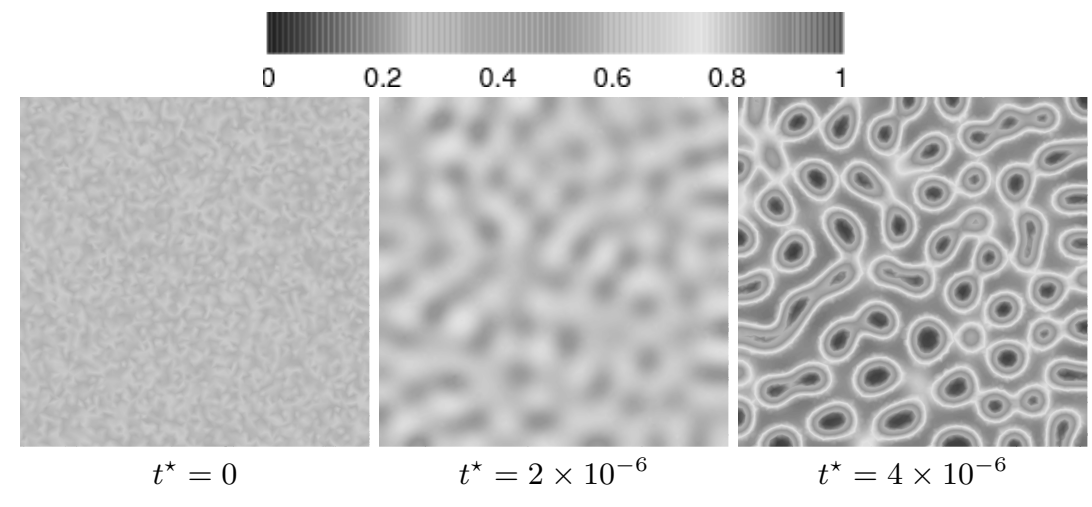

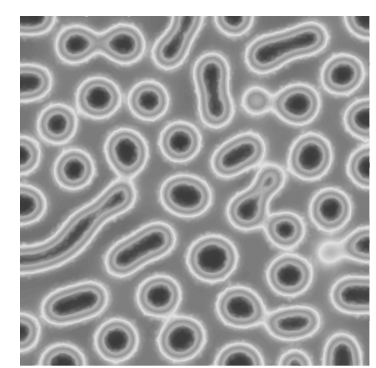

$t^{\star}=8 \times 10^{-6}$

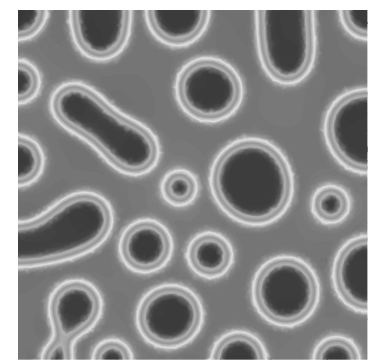

$t^{\star}=6.4 \times 10^{-5}$

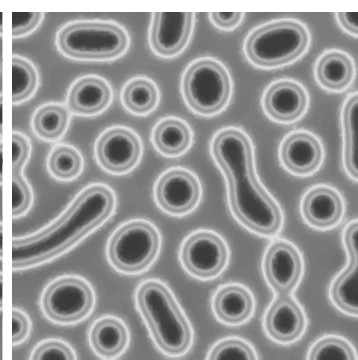

$t^{\star}=1.6 \times 10^{-5}$

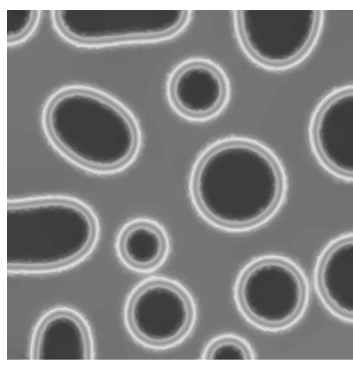

$t^{\star}=1.28 \times 10^{-4}$

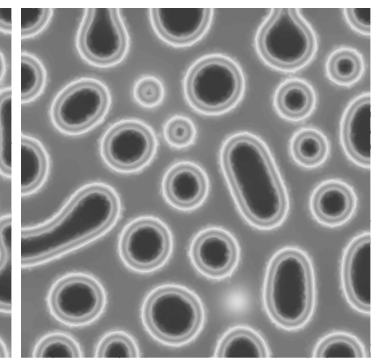

$t^{\star}=3.2 \times 10^{-5}$

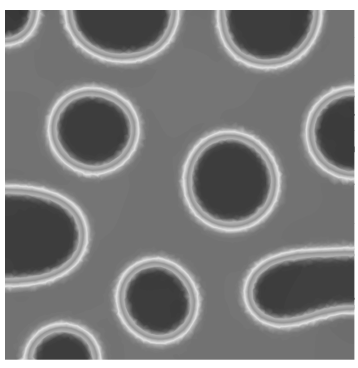

$t^{\star}=2.56 \times 10^{-4}$

Fig. 1. Evolution of concentration contours from a randomly perturbed initial condition.

Using a random triangulation with $h \approx 1 / 100$, the evolution of the concentration field is depicted in Figure 1. The concentration evolution can basically be categorised in two phases: the first phase, which is predominantly governed by spinodal decomposition and phase separation, and a second phase which is characterised by grain coarsening. During the first phase, changes in concentration are driven primarily by the minimisation of the local chemical energy $\Psi^{c}$. This period is basically terminated as soon as the local concentration is driven to either value of the two binodal points. Approximately from 
$t^{\star}=8 \times 10^{-6}$ onwards, local changes in concentrations are primarily governed by the surface free energy $\Psi^{s}$. In order to minimise its contribution, the generated patterns cluster and grains tend to coarsen. This Ostwald ripening takes place at a much longer time scale.

\section{Conclusions}

A fully discrete finite element formulation for the Cahn-Hilliard equation has been analysed. The formulation requires consideration of the concentration field only and exploits simple Lagrange finite element basis functions. The necessary continuity of derivatives across element boundaries is enforced in a weak sense and a penalty term acting on jumps in the normal derivative across element boundaries is added to maintain stability.

It is shown for Crank-Nicolson and backward Euler time stepping schemes that critical time steps for both uniqueness and stability exist, but are independent on the element size $h$. Interestingly, the allowable time step is related to the penalty term. A larger penalty term enhances stability and allows for a larger time step. It is possible that the presented time step restrictions could be tightened and the time step restriction for uniqueness quantified. For the explicit forward Euler scheme, the critical time step scales with $h^{4}$, making the scheme impractical. Finally, the fully discrete problem has been posed in such a form that an existing a priori error estimate for the Cahn-Hilliard equation in the $L^{2}$ norm can be applied directly, proving optimal rates of convergence for the proposed scheme.

\section{Acknowledgements}

GNW was supported by the Netherlands Technology Foundation STW, applied science division of NWO and the technology programme of the Ministry of Economic Affairs. KG was supported by a Presidential Early Career Award for Scientists and Engineers through the US Department of Energy.

\section{References}

1. Cahn JW, Hilliard JE (1958) Free energy of a nonuniform system-I: Interfacial free energy. The Journal of Chemical Physics 28(2):258-267.

2. Elliott CM, Zheng S (1986) On the Cahn-Hilliard Equation. Archive for Rational Mechanics and Analysis 96(4):339-357.

3. Elliott CM, French DA (1987) Numerical studies of the Cahn-Hilliard equation for phase separation. IMA Journal of Applied Mathematics 38:97-128. 
4. Elliott CM, French DA (1989) A non-conforming finite element method for the two-dimensional Cahn-Hilliard equation. SIAM Journal on Numerical Analysis 26(4):884-903.

5. Elliott CM, French DA, Milner FA (1989) A 2nd-order splitting method for the Cahn-Hilliard equation. Numerische Mathematiek 54(5):575-590.

6. Du Q, Nicolaides RA (1991) Numerical analysis of a continuum model of phase transition. SIAM Journal on Numerical Analysis 28(5):1310-1322.

7. Copetti MIM, Elliot CM (1992) Numerical analysis of the Cahn-Hilliard equation with a logarithmic free energy. Numerische Mathematik 63(1):39-65.

8. Blowey JF, Elliott CM (1992) The Cahn-Hilliard gradient theory for phase separation with non-smooth free energy Part II: Numerical analysis. European Journal of Applied Mathematics 3:147-179.

9. Wells GN, Kuhl E, Garikipati K (2006) A discontinuous Galerkin formulation for the Cahn-Hilliard equation. Journal of Computational Physics $218(2): 860-877$.

10. Engel G, Garikipati K, Hughes TJR, Larson MG, Mazzei L, Taylor RL (2002) Continuous/discontinuous finite element approximations of fourthorder elliptic problems in structural and continuum mechanics with applications to thin beams and plates, and strain gradient elasticity. Computer Methods in Applied Mechanics and Engineering 191(34):3669-3750. 